\title{
The Change in Body Weight During Hospitalization Predicts Mortality in Patients With Acute Decompensated Heart Failure
}

\author{
Tomo Komakia, b, Shin-ichiro Miura ${ }^{\mathrm{a}, \mathrm{c}, \mathrm{d}}$, Tadaaki Arimura ${ }^{\mathrm{a}}$, Yuhei Shiga ${ }^{\mathrm{a}}$, Joji Morii ${ }^{\mathrm{a}}$, \\ Takashi Kuwano ${ }^{\text {a }}$, Satoshi Imaizumi ${ }^{\text {a }}$, Ken Kitajima ${ }^{a}$, Atsushi Iwata ${ }^{\text {a }}$, Natsumi Morito ${ }^{\text {a }}$, \\ Eiji Yahiro $^{a}$, Kanta Fujimi ${ }^{a}$, Akira Matsunaga ${ }^{b}$, Keijiro Saku ${ }^{\mathrm{a}, \mathrm{c}}$
}

\begin{abstract}
Background: In our experience, the change in body weight (BW) during hospitalization varies greatly in patients with acute decompensated heart failure (HF). Since the clinical significance of a change in BW is not clear, we investigated whether a change in BW could predict mortality.
\end{abstract}

Methods: We retrospectively enrolled 130 patients (72 males; aged $68 \pm 10$ years) who were hospitalized due to acute decompensated $\mathrm{HF}$ and followed for 2 years after discharge. The change in the BW index during hospitalization $(\triangle \mathrm{BWI})$ was calculated as (BW at hospital admission minus BW at hospital discharge)/body surface area at hospital discharge.

Results: The patients were divided into quartiles according to $\Delta \mathrm{BWI}$, and the 2-year mortality rates in the quartiles with the lowest, second, third and highest $\triangle \mathrm{BWI}$ were $18.8 \%, 12.1 \%, 3.1 \%$ and $9.1 \%$, respectively. In a multivariate Cox proportional hazards analysis after adjusting for variables with a $\mathrm{P}$ value less than $0.05, \Delta \mathrm{BWI}$ was independently associated with 2 -year mortality $(\mathrm{P}=0.0002)$, and the quartile with the lowest $\triangle \mathrm{BWI}$ had a higher relative risk (RR) for 2-year mortality than the quartile with the highest $\triangle \mathrm{BWI}$ (RR: 7.46, 95\% confidence interval: $1.03-53.99, \mathrm{P}=0.04$ ).

Conclusion: In conclusion, $\Delta \mathrm{BWI}$ was significantly associated with 2-year mortality after discharge, which indicates that $\Delta \mathrm{BWI}$ might be a simple predictor of prognosis in acute decompensated HF.

Keywords: Predictor of prognosis; Change in body weight index; Acute decompensated heart failure

Manuscript accepted for publication January 10, 2017

aDepartment of Cardiology, Fukuoka University School of Medicine, Fukuoka, Japan

${ }^{b}$ Department of Laboratory Medicine, Fukuoka University School of Medicine, Fukuoka, Japan

'Department of Molecular Cardiovascular Therapeutics, Fukuoka University School of Medicine, Fukuoka, Japan

${ }^{\mathrm{d} C o r r e s p o n d i n g ~ A u t h o r: ~ S h i n-i c h i r o ~ M i u r a, ~ D e p a r t m e n t ~ o f ~ C a r d i o l o g y, ~ F u k u o-~}$ ka University School of Medicine, 7-45-1 Nanakuma, Jonan-ku, Fukuoka 814-0180, Japan. Email: miuras@cis.fukuoka-u.ac.jp

doi: https://doi.org/10.14740/jocmr2890w

\section{Introduction}

Heart failure (HF) is a clinical syndrome that occurs in patients who, because of an inherited or acquired abnormality of cardiac structure and/or function, develop a constellation of clinical symptoms (dyspnea and fatigue) and signs (edema and rales). Patients with HF are repeatedly hospitalized and experience a poor quality of life and a shortened life expectancy. Although long-term mortality rates for patients with HF are gradually improving through the application of various therapies [1-7], they are still unacceptably high [8]. In recent years, there has been an increase in the number of biomarkers in HF, such as troponin [9], estimated glomerular filtration rate [10], cystatin C [11], serum uric acid [12], and anemia [13], in addition to B-type natriuretic peptide (BNP) and amino acid N-terminal pro-BNP $[14,15]$. The ultimate clinical role of these novel biomarkers is not clear. Since patients with HF often show fluid retention with leg edema regardless of the etiology of HF, an important goal in the acute phase of HF is fluid control by diuretics. When we treated patients with HF in clinical practice, we noted that the change in body weight (BW) during hospitalization varied greatly in patients with acute decompensated HF. We considered that the change in BW during hospitalization may be a simple predictor of the prognosis in HF. There have been no previous reports on the association between changes in BW due to treatment and the prognosis of HF. The patients with greater changes in BW may have a wide range of therapeutic responses, compared to those with smaller changes who exhibit a narrow range of responses. Therefore, we hypothesized that patients with greater changes in BW might have a better prognosis than those with smaller changes.

In this study, we investigated the associations between the change in BW during hospitalization and the prognosis in patients with acute decompensated HF.

\section{Methods}

\section{Study population}

We retrospectively investigated the association between the change in $\mathrm{BW}$ during hospitalization and the prognosis of HF. 
Table 1. Patient Characteristics in the Survival and Death Groups

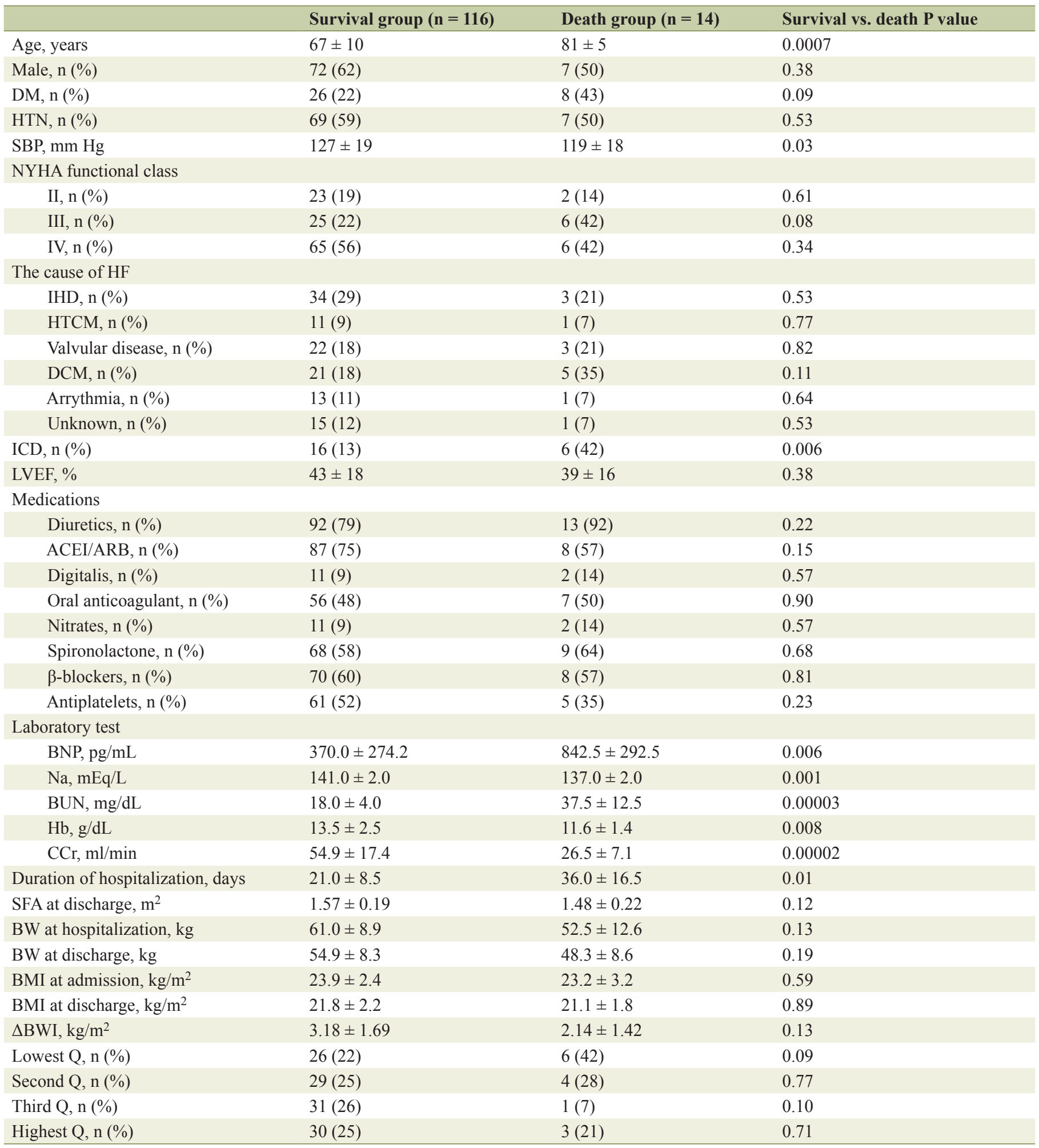

DM: diabetes mellitus; HTN: hypertension; SBP: systolic blood pressure; NYHA: New York Heart Association; HF: heart failure; IHD: ischemic heart disease; HTCM: hypertensive cardiomyopathy; DCM: dilated cardiomyopathy; ICD: implantable cardioverter defibrillator; LVEF: left ventricular ejection fraction; ACEI: angiotensin converting enzyme inhibitors; ARB: angiotensin II type 1 receptor blocker; BNP: brain natriuretic peptide; Na: sodium; BUN: blood urea nitrogen; $\mathrm{Hb}$ : hemoglobin; $\mathrm{CCr}$ : creatinine clearance; SFA: surface area; BW: body weight; BMI: body mass index; $\triangle B \mathrm{Bl}$ : changes in body weight index; Q: quartile. 
We enrolled 130 consecutive patients who were initially hospitalized due to acute decompensated HF at Fukuoka University Hospital from 2001 to 2013 and followed for 2 years after discharge. The diagnosis of HF was established by the simultaneous presence of at least two major Framingham criteria or one major criterion in conjunction with two minor criteria. The primary end-point was 2-year mortality after discharge. We excluded patients with end-stage renal disease under maintenance dialysis. This study was approved by the Ethics Committee of Fukuoka University Hospital (\#16-1-21). We collected and analyzed all data using the database of Fukuoka University Hospital.

\section{Clinical parameters}

Data on age, gender, BW at admission and discharge, body mass index (BMI) at admission and discharge, systolic blood pressure (SBP) at admission, body surface area (SFA) at discharge calculated by the formula of DuBois, duration of hospitalization, New York Heart Association (NYHA) functional class at hospitalization, the etiology of HF, the presence or absence of diabetes mellitus (DM) and hypertension (HTN), medications, left ventricular ejection fraction (LVEF) using Simpson's method, BNP, hemoglobin ( $\mathrm{Hb})$, serum sodium, blood urea nitrogen (BUN), creatinine clearance (CCr) calculated by the formula of Cockcroft-Gault, and the presence of an implantable cardioverter defibrillator (ICD) were collected. In the measurement of BW at admission, a weighing scale was used for mobile patients with acceptable dyspnea, while a weighing and lifting scale was used in immobile patients with severe dyspnea. The change in the $\mathrm{BW}$ index $(\Delta \mathrm{BWI})$ during hospitalization was calculated as (BW at hospital admission minus BW at hospital discharge)/(SFA at hospital discharge).

The cause of HF was classified as ischemic heart disease (IHD), hypertensive cardiomyopathy (HTCM), valvular heart disease, dilated cardiomyopathy (DCM), arrhythmia, or unknown. When there were multiple causes of HF, the main cause of HF was assumed based on the patient's medical history. Patients who had a current SBP and DBP $\geq 140$ and 90 $\mathrm{mm} \mathrm{Hg}$, respectively, or who were receiving antihypertensive therapy were considered to have HTN. DM was defined using the Japan Diabetes Society criteria.

\section{Statistical analysis}

All statistical analyses were performed using StatFlex V6 (Artech Co., Ltd., Osaka, Japan). Date were expressed as the mean \pm standard deviation (SD) or median \pm quartile deviation (QD) for continuous variables and as a percentage (\%) for categorical variables. Subjects were classified into two groups according to 2-year mortality (survival and death groups) after discharge. In addition, subjects were categorized into quartiles according to $\Delta \mathrm{BWI}$ as follows: $<1.3 \mathrm{~kg} / \mathrm{m}^{2}$ (lowest $\Delta \mathrm{BWI}$ ), $1.3-2.9 \mathrm{~kg} / \mathrm{m}^{2}$ (second $\Delta \mathrm{BWI}$ ), $3-4.7 \mathrm{~kg} / \mathrm{m}^{2}$ (third $\Delta \mathrm{BWI}$ ) and $\geq 4.8 \mathrm{~kg} / \mathrm{m}^{2}$ (highest $\Delta \mathrm{BWI}$ ). Categorical and continuous variables were compared between two groups by a
Chi-square analysis and Student's $t$-test, respectively. When continuous variables did not show a normal distribution, we performed a Mann-Whitney $U$ test. Categorical and continuous variables were compared among the quartile $\Delta \mathrm{BWI}$ groups by a Chi-square analysis and one-way analysis of variance, respectively. When continuous variables did not show a normal distribution, we performed a Kruskal-Wallis test. The univariate relationship between $\triangle \mathrm{BWI}$ and 2-year mortality was evaluated using a Kaplan-Meier analysis. Differences in 2-year mortality across the quartiles of $\Delta \mathrm{BWI}$ were evaluated using the log-rank test. The association between $\Delta \mathrm{BWI}$ and 2-year mortality was evaluated by Cox proportional hazards regression. Before the multivariate analysis, the duration of hospitalization, age, $\mathrm{CCr}$, BW at admission and discharge, $\mathrm{BMI}$ at admission and discharge, SBP at admission, BUN, serum sodium, BNP and $\triangle \mathrm{BWI}$ were normalized by a power transformation to correct for a skewed distribution. A backward-stepwise Cox proportional hazards model was used to identify the independent predictors of mortality, retaining variables with $\mathrm{P}<0.05$. Significant factors included the etiology of HTCM, DM, CCr, SBP at admission, BNP, $\beta$-blockers, duration of hospitalization, and $\triangle \mathrm{BWI}$. Additional covariates were not considered to be independent predictors of mortality, including age, gender, HTN, DM, NYHA functional class, $\mathrm{Hb}$, serum sodium, BUN, the etiology of IHD, valvular cardiac disease and arrhythmia, LVEF, the use of diuretics, angiotensin converting enzyme inhibitors (ACEI) or angiotensin II type 1 receptor blocker (ARB), digitalis, oral anticoagulants, nitrates, spironolactone and antiplatelets, $\mathrm{BW}$ at admission and discharge, BMI at admission and discharge, and the presence of ICD implantation. A multivariate Cox proportional hazards analysis was then conducted, and the above variables were combined with the categorized $\Delta \mathrm{BWI}$. The reference group was defined as $\Delta \mathrm{BWI} \geq 4.8 \mathrm{~kg} / \mathrm{m}^{2}$, which was the quartile with the highest $\Delta \mathrm{BWI}$. $\mathrm{P}<0.05$ was considered to be statistically significant.

\section{Results}

\section{Patient characteristics in the survival and death groups}

The mean age of the 130 patients was $68 \pm 10$ years, and 79 of them were male $(60 \%)$. Fourteen patients died during the 2-year follow-up period (11\%). The cause of death was progressive pump failure in 13 patients and sudden death in one and six of these patients were implanted with an ICD (42\%). Table 1 shows the patient characteristics in the survival $(\mathrm{n}=$ $116)$ and death $(\mathrm{n}=14)$ groups. The patients in the death group were significantly older and had lower SBP at admission, lower $\mathrm{CCr}, \mathrm{Hb}$ and serum sodium, higher BNP and BUN, a longer duration of hospitalization and more frequent ICD implantation than those in the survival group.

\section{Mortality rates in quartiles according to $\triangle \mathrm{BWI}$}

The mortality rates in quartiles according to $\triangle \mathrm{BWI}$ were $19 \%$ 


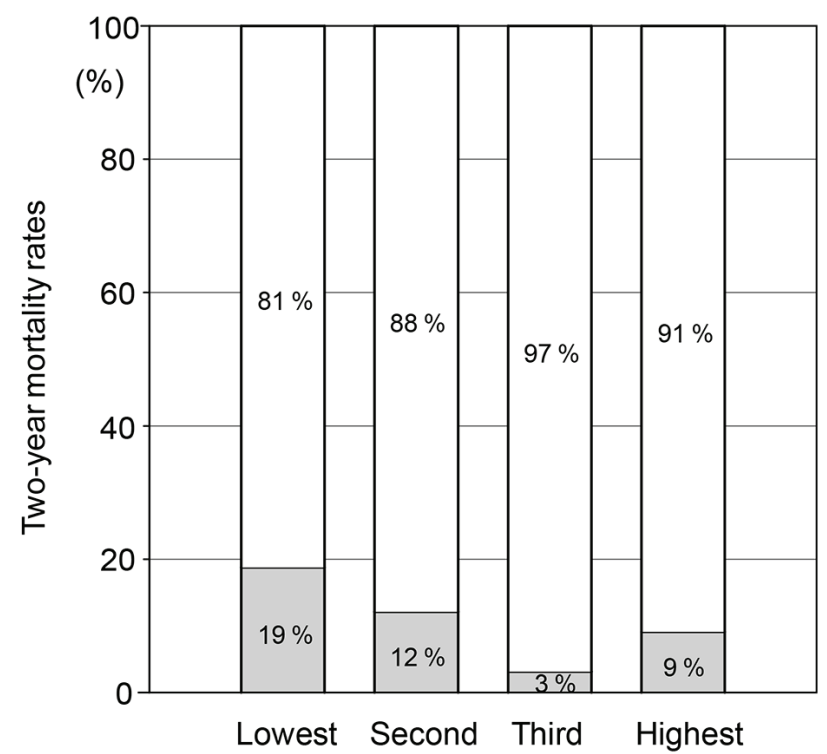

Figure 1. Mortality rates in quartiles (lowest, second, third and highest) according to $\triangle \mathrm{BWI}$.

$(\mathrm{n}=6), 12 \%(\mathrm{n}=4), 3 \%(\mathrm{n}=1)$, and $9 \%(\mathrm{n}=3)$ in the quartiles with the lowest, second, third and highest $\triangle \mathrm{BWI}$, respectively (Fig. 1).

\section{Patient characteristics in quartiles according to $\Delta \mathrm{BWI}$}

The patient characteristics in the quartiles of $\triangle \mathrm{BWI}$ are shown in Table 2. In a comparative analysis, age, gender, NYHA functional class II and IV, LVEF, BW at admission and duration of hospitalization significantly differed among the quartiles of $\triangle \mathrm{BWI}$.

\section{Kaplan-Meier analysis of survival in the quartiles of $\Delta \mathrm{BWI}$}

Figure 2 shows the Kaplan-Meier curves of survival in the quartiles of $\triangle B W I$. Patients were categorized into quartiles according to $\triangle \mathrm{BWI}$. In unadjusted comparisons, the thirdquartile $\Delta \mathrm{BWI}$ group had the best survival, while the quartile with the lowest $\Delta \mathrm{BWI}$ had the worst survival. A log-rank test showed that the difference between the quartiles with the third and lowest $\triangle \mathrm{BWI}$ was statistically significant $(\mathrm{P}=$ $0.045)$.

\section{Cox proportional hazards analyses to identify independent predictors of mortality}

In the adjusted Cox proportional hazards analyses, as shown in Table 3 , a decrease in $\triangle B W I$ was found to be a statistically significant predictor of 2-year mortality, as were other variables including SBP at admission, BNP, DM, CCr, duration of hospitalization, use of $\beta$-blocker and the etiology of HTCM.

\section{A multivariate Cox proportional hazards analysis com- bined with categorized $\Delta \mathrm{BWI}$}

In the quartiles according to $\triangle \mathrm{BWI}$, the quartile with the lowest $\triangle B W I$ showed a much higher risk of 2-year mortality than the quartile with the highest $\triangle \mathrm{BWI}$ (Table 4) (relative risk: 7.46, 95\% CI: 1.03 - 53.99, $\mathrm{P}=0.04)$.

\section{Discussion}

The main finding in this study was that $\Delta \mathrm{BWI}$ in hospitalized patients with acute decompensated HF was significantly associated with 2-year mortality after discharge independent of various covariates. The quartile with the lowest $\triangle B W I$ showed a much higher risk of 2-year mortality than that with the highest $\Delta \mathrm{BWI}$ as assessed by a multivariate Cox proportional hazards analysis. Therefore, we should reinforce the treatment of HF to the patients with low $\triangle B W I$, such as with additional medications, cardiac rehabilitation, Waon therapy, the introduction of supplemental oxygen therapy, and cardiac resynchronization therapy.

In this study, we first hypothesized that patients with a greater change in BW may have a better prognosis. Our findings support this hypothesis. Many previous reports have identified univariate predictors of reduced survival, including NYHA functional class, reduced LVEF [16], renal insufficiency, and comorbid factors, such as DM [17] and IHD. Predictive models that consider comorbidities and HF-related factors, such as the EFFECT model [18], the HF survival score [19], and the Seattle Heart Failure Model [20] have also been shown to be associated with the prognosis. However, these previous predictors and models have been insufficient: the former are limited in their ability to predict survival in individual patients, and the latter are complicated and are not performed in all institutions because of multiple factors, including the need for invasive tests. Since $\triangle B W I$ reflects the cardio-renal connection, it can be used in individual patients. Additionally, it can be used in all institutions because it is simple, inexpensive and easy to measure.

Longer period of hospitalization, lower SBP at admission, higher BNP, lower $\mathrm{CCr}$ and \% $\beta$-blockers were also associated with 2-year mortality, as shown in Table 3 , and these associations were consistent with previous reports [7, 8, 15, 21, 22]. Thus, the results indicated that the patients in this study did not represent any specific patient group.

This study has several limitations. First, this study was limited by its retrospective design. Although this study considered only a small number of patients, we collected data that we could follow for up to 2 years in as many patients as possible in our hospital from 2001 to 2013. The medication strategies in patients with HF, such as with respect to the use of human atrial natriuretic peptide and $\beta$-blockers, in the 1990s were much different from those used presently. Thus, the use of older data may directly influence the mortality. Second, the analysis was performed under conditions of various etiologies of $\mathrm{HF}$ and various medications. To confirm the clinical significance of the relationship between $\triangle \mathrm{BWI}$ and 2-year mortality, we performed a multivariate Cox proportional hazards analysis. Prospective studies will be needed to clarify these limitations. 
Table 2. Patient Characteristics in Quartiles According to $\triangle \mathrm{BWI}$

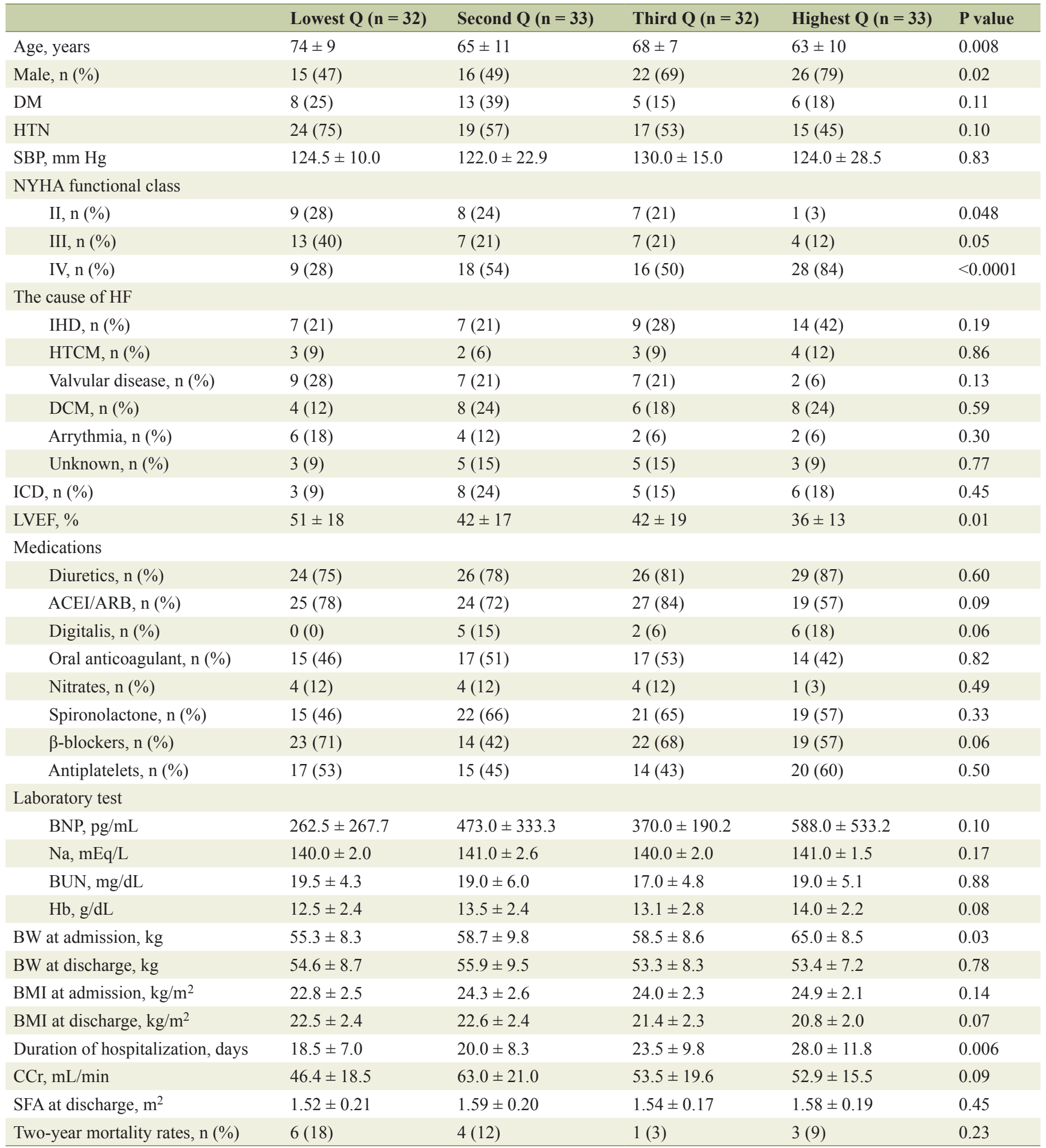

$\triangle B W I$ : changes in body weight index; Q: quartile; DM: diabetes mellitus; HYN; hypertension; SBP: systolic blood pressure; NYHA: New York Heart Association; HF; heart failure; IHD: ischemic heart disease; HTCM: hypertensive cardiomyopathy; DCM: dilated cardiomyopathy; ICD: implantable cardioverter defibrillator; LVEF: left ventricular ejection fraction; ACEI: angiotensin converting enzyme inhibitors; ARB: angiotensin II type 1 receptor blocker; BNP: brain natriuretic peptide; Na: sodium; BUN: blood urea nitrogen; Hb: hemoglobin; BW: body weight; BMI: body mass index; CCr: creatinine clearance; SFA: surface area. 


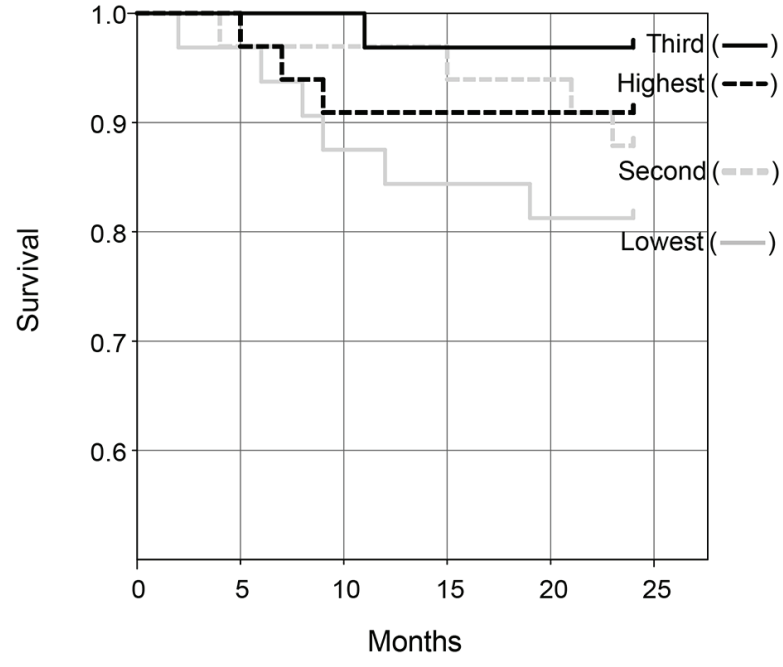

Figure 2. Kaplan-Meier curves of survival in quartiles (lowest, second, third and highest) according to $\triangle \mathrm{BWI}$.

In conclusion, $\Delta \mathrm{BWI}$ was significantly associated with 2-year mortality after discharge, which indicates that $\triangle \mathrm{BWI}$ might be a simple predictor of prognosis in acute decompensated HF.

\section{Conflicts of Interest}

KS is a Chief Director and SM is a Director of NPO Clinical and Applied Science, Fukuoka, Japan. KS has an Endowed "Department of Molecular Cardiovascular Therapeutics" supported by MSD, Co. LTD. SM belongs to the Department of Molecular Cardiovascular Therapeutics, which is supported by MSD, Co. LTD.

\section{References}

1. Effect of enalapril on survival in patients with reduced left ventricular ejection fractions and congestive heart

Table 3. Cox Proportional Hazards Analyses to Identify Independent Predictors of Mortality

\begin{tabular}{llll}
\hline & $\boldsymbol{\beta}$ & z value & P value \\
\hline DM & 2.51 & 3.05 & 0.0023 \\
Duration of hospitalization & 0.99 & 3.38 & 0.0007 \\
CCr & -1.01 & 3.7 & 0.0002 \\
\hline$\Delta$ BWI & -3.53 & 3.72 & 0.0002 \\
$\beta$-blockers & -3.66 & 2.97 & 0.0029 \\
HTCM & 5.53 & 3.06 & 0.0022 \\
BNP & 0.52 & 3.44 & 0.0006 \\
SBP & -1.29 & 3.67 & 0.0002 \\
\hline
\end{tabular}

DM: diabetes mellitus; $C C r$ : creatinine clearance; $\triangle \mathrm{BWI}$ : changes in body weight index; HTCM: hypertensive cardiomyopathy; BNP: brain natriuretic peptide; SBP: systolic blood pressure.
Table 4. A Multivariate Cox Proportional Hazards Analysis Combined With Categorized $\Delta \mathrm{BWI}$

\begin{tabular}{lllll}
\multicolumn{1}{r}{} & $\boldsymbol{\beta}$ & z value & P value & RR $(\mathbf{9 5 \%}$ CI) \\
\hline$\Delta$ BWI & & & & \\
Lowest Q & 2 & 1.99 & 0.04 & $7.46(1.03-53.99)$ \\
Second Q & 1.39 & 1.6 & 0.1 & $4.02(0.73-22.04)$ \\
Third Q & -0.1 & 0.08 & 0.93 & $0.89(0.07-11.16)$ \\
Highest Q & & & & 1.00 \\
\hline
\end{tabular}

$\triangle \mathrm{BWI}$ : changes in body weight index; $\mathrm{Q}$ : quartile; RR: relative risk; $\mathrm{Cl}$ : confidence interval.

failure. The SOLVD Investigators. $N$ Engl $\mathrm{J}$ Med. 1991;325(5):293-302.

2. Granger CB, McMurray JJ, Yusuf S, Held P, Michelson EL, Olofsson B, Ostergren J, et al. Effects of candesartan in patients with chronic heart failure and reduced leftventricular systolic function intolerant to angiotensinconverting-enzyme inhibitors: the CHARM-Alternative trial. Lancet. 2003;362(9386):772-776.

3. Pitt B, Zannad F, Remme WJ, Cody R, Castaigne A, Perez A, Palensky J, et al. The effect of spironolactone on morbidity and mortality in patients with severe heart failure. Randomized Aldactone Evaluation Study Investigators. N Engl J Med. 1999;341(10):709-717.

4. Zannad F, McMurray JJ, Krum H, van Veldhuisen DJ, Swedberg K, Shi H, Vincent J, et al. Eplerenone in patients with systolic heart failure and mild symptoms. $\mathrm{N}$ Engl J Med. 2011;364(1):11-21.

5. Bristow MR, Gilbert EM, Abraham WT, Adams KF, Fowler MB, Hershberger RE, Kubo SH, et al. Carvedilol produces dose-related improvements in left ventricular function and survival in subjects with chronic heart failure. MOCHA Investigators. Circulation. 1996;94(11):28072816.

6. Uemura Y, Shibata R, Takemoto K, Uchikawa T, Koyasu M, Ishikawa S, Mitsuda T, et al. Clinical benefit of tolvaptan in patients with acute decompensated heart failure and chronic kidney disease. Heart Vessels. 2016;31(10):16431649.

7. Nabeta T, Inomata T, Iida Y, Ikeda Y, Iwamoto-Ishida M, Ishii S, Naruke T, et al. Prognostic significance of betablocker up-titration in conjunction with cardiac resynchronization therapy in heart failure management. Heart Vessels. 2016;31(7):1109-1116.

8. Ho KK, Pinsky JL, Kannel WB, Levy D. The epidemiology of heart failure: the Framingham Study. J Am Coll Cardiol. 1993;22(4 Suppl A):6A-13A.

9. Yancy CW, Jessup M, Bozkurt B, Butler J, Casey DE, Jr., Drazner MH, Fonarow GC, et al. 2013 ACCF/AHA guideline for the management of heart failure: a report of the American College of Cardiology Foundation/American Heart Association Task Force on practice guidelines. Circulation. 2013;128(16):e240-327.

10. Halkar M, Tang WH. Incorporating common biomarkers into the clinical management of heart failure. Curr Heart Fail Rep. 2013;10(4):450-457. 
11. Dupont M, Wu Y, Hazen SL, Tang WH. Cystatin C identifies patients with stable chronic heart failure at increased risk for adverse cardiovascular events. Circ Heart Fail. 2012;5(5):602-609.

12. Vaduganathan M, Greene SJ, Ambrosy AP, Mentz RJ, Subacius HP, Chioncel O, Maggioni AP, et al. Relation of serum uric acid levels and outcomes among patients hospitalized for worsening heart failure with reduced ejection fraction (from the efficacy of vasopressin antagonism in heart failure outcome study with tolvaptan trial). Am J Cardiol. 2014;114(11):1713-1721.

13. Felker GM, Allen LA, Pocock SJ, Shaw LK, McMurray JJ, Pfeffer MA, Swedberg K, et al. Red cell distribution width as a novel prognostic marker in heart failure: data from the CHARM Program and the Duke Databank. J Am Coll Cardiol. 2007;50(1):40-47.

14. Januzzi JL, Jr., Camargo CA, Anwaruddin S, Baggish AL, Chen AA, Krauser DG, Tung R, et al. The N-terminal ProBNP investigation of dyspnea in the emergency department (PRIDE) study. Am J Cardiol. 2005;95(8):948-954.

15. Nakada Y, Takahama H, Kanzaki H, Sugano Y, Hasegawa T, Ohara T, Amaki M, et al. The predictability of reninangiotensin-aldosterone system factors for clinical outcome in patients with acute decompensated heart failure. Heart Vessels. 2016;31(6):925-931.

16. Wong M, Staszewsky L, Latini R, Barlera S, Glazer R, Aknay N, Hester A, et al. Severity of left ventricular remodeling defines outcomes and response to therapy in heart failure: Valsartan heart failure trial (Val-HeFT) echocar- diographic data. J Am Coll Cardiol. 2004;43(11):20222027.

17. Dries DL, Sweitzer NK, Drazner MH, Stevenson LW, Gersh BJ. Prognostic impact of diabetes mellitus in patients with heart failure according to the etiology of left ventricular systolic dysfunction. J Am Coll Cardiol. 2001;38(2):421-428.

18. Lee DS, Austin PC, Rouleau JL, Liu PP, Naimark D, Tu JV. Predicting mortality among patients hospitalized for heart failure: derivation and validation of a clinical model. JAMA. 2003;290(19):2581-2587.

19. Aaronson KD, Schwartz JS, Chen TM, Wong KL, Goin JE, Mancini DM. Development and prospective validation of a clinical index to predict survival in ambulatory patients referred for cardiac transplant evaluation. Circulation. 1997;95(12):2660-2667.

20. Levy WC, Mozaffarian D, Linker DT, Sutradhar SC, Anker SD, Cropp AB, Anand I, et al. The Seattle Heart Failure Model: prediction of survival in heart failure. Circulation. 2006;113(11):1424-1433.

21. Smith GL, Lichtman JH, Bracken MB, Shlipak MG, Phillips CO, DiCapua P, Krumholz HM. Renal impairment and outcomes in heart failure: systematic review and meta-analysis. J Am Coll Cardiol. 2006;47(10):1987-1996.

22. Gheorghiade M, Abraham WT, Albert NM, Greenberg BH, O'Connor CM, She L, Stough WG, et al. Systolic blood pressure at admission, clinical characteristics, and outcomes in patients hospitalized with acute heart failure. JAMA. 2006;296(18):2217-2226. 